\title{
Co-planar light-actuated optoelectrowetting microfluidic device for droplet manipulation
}

\author{
Jodi Loo $\odot$,* Shao Ning Pei, and Ming C. Wu $\odot$ \\ University of California, Berkeley, Electrical Engineering and Computer Sciences Department, \\ Berkeley, California, United States
}

\begin{abstract}
We report on a co-planar light-actuated digital microfluidics device that features a metal mesh grid integrated on the device surface to allow droplets to be exposed from above. We discuss a theoretical circuit model for our co-planar optoelectrowetting (OEW) design that allows for the optimization of droplet actuation while maintaining reliable droplet movement. Basic droplet manipulations such as merging and parallel actuation of droplets are achieved at speeds of up to $4.5 \mathrm{~cm} / \mathrm{s}$. The co-planar OEW device design benefits from having an open top design that allows for a wider range of system integration configurations than previous generations of OEW devices. A droplet-on-demand dispensing system from above is integrated with the co-planar OEW device to demonstrate the versatility of this optofluidic platform. The ability to inject, collect, and position individual droplets to form large-scale droplet arrays of up to $20 \times$ 20 is achieved. (C) The Authors. Published by SPIE under a Creative Commons Attribution 4.0 Unported License. Distribution or reproduction of this work in whole or in part requires full attribution of the original publication, including its DOI. [DOI: 10.1117/1.JOM.1.3.034001]
\end{abstract}

Keywords: optoelectrowetting; optofluidics; light-actuated digital microfluidics; droplet microfluidics.

Paper 21005 received Mar. 8, 2021; accepted for publication Apr. 28, 2021; published online May 12, 2021.

\section{Introduction}

Droplet-based digital microfluidics addresses many requirements for lab-on-a-chip systems through the ability to process a large number of samples using reduced sample and reagent volumes while increasing detection sensitivity. Digital microfluidics has seen much development over the last couple of decades ${ }^{1-7}$ with an increase in applications utilizing digital microfluidic technologies such as synthetic biology,${ }^{8}$ single cell analysis, ${ }^{9,10}$ proteomic analysis, ${ }^{11}$ genomic sequencing, ${ }^{12}$ and diagnostics. ${ }^{13}$ As many of these technologies are becoming further developed and commercialized, the range of applications that digital microfluidics can tackle continues to grow.

Droplet microfluidics can be categorized into two different types. The first is individual waterin-oil emulsions that flow through microfluidic channels such as in the continuous flow microfluidic paradigm. ${ }^{14-19}$ This type of droplet microfluidics benefits from its high throughput ability to rapidly produce water-in-oil emulsions. However, these droplets must be processed sequentially as customizable operations for unique droplets are not feasible. The second type of digital microfluidics is an individually addressable droplet system. This type encompasses several different technologies such as systems based on dielectrophoresis, ${ }^{20,21}$ acoustic waves, ${ }^{22-24}$ magnetic beads, ${ }^{25-27}$ electrowetting on dielectric, ${ }^{1,2,28}$ and optoelectrowetting (OEW) ${ }^{29}$ Compared with droplet microfluidics by continuous flow microfluidics where mechanical on-chip pressure components such as pumps and valves help control fluid flow in channels, ${ }^{30,31}$ individually addressed droplet microfluidics allows discrete droplets to move independently around the plane of the device. Droplets are often actuated by electrical, optical, or magnetic means.

OEW enables parallel manipulation of a large number of droplets using projected light images based on the principles of light-controlled electrowetting. ${ }^{29,32-36}$ Previously, OEW devices for picoliter to microliter sized droplet manipulation have been achieved. ${ }^{33}$ These traditional OEW devices also provide a flexible platform to perform chemical and biological assays such as

*Address all correspondence to Jodi Loo, jodiloo@eecs.berkeley.edu 
real-time isothermal polymerase chain reaction with basic droplet manipulation techniques. ${ }^{35,36}$ However, one shortcoming of the traditional OEW device is its limitation in designing input and output configurations for larger microfluidic system integration. For previously reported OEW devices, droplets are sandwiched between a bottom active OEW substrate and a top layer ground electrode substrate. Due to the top cover electrode, droplets are sandwiched between the active OEW device and the top cover, forcing any droplet dispensing or extraction techniques to be designed from the side openings. Although feasible, this can prove to be challenging. Exposed fluidic droplets from above allows for easier access as well as more fluidic operations and integration schemes. Although single-sided OEW devices have been reported, these designs are less efficient as they require very high voltages and light intensities for operation due to a greatly reduced OEW force. ${ }^{37,38}$

In this paper, we report on a co-planar OEW device that eliminates the need for a top cover electrode through the implementation of a conductive metal grid on the OEW surface while also benefiting from maintaining a high OEW force. The co-planar OEW device can be operated under the same reduced voltage and low-intensity light conditions as the two-plated traditional OEW device with over $2 \times$ faster droplet speeds. Sessile droplets are no longer sandwiched between the main OEW device surface and the top electrode cover and are now accessible from above in addition to the sides. Droplets move freely around the two-dimensional plane as movement is not limited to the grid pattern itself. This configuration allows for direct droplet access for microfluidic system integration and droplet dispensing and extraction.

\section{Device Design and Methods}

\subsection{Device Design}

The co-planar OEW device structure is depicted in Fig. 1. The device is made from an indiumtin-oxide (ITO)-coated glass substrate with an amorphous silicon (a-Si) photoconductive layer and an aluminum oxide $\left(\mathrm{Al}_{2} \mathrm{O}_{3}\right)$ dielectric layer deposited on top. The co-planar OEW device has an integrated metal mesh ground network that is fabricated on top of the dielectric layer. A droplet is placed on the surface of the co-planar OEW device above the metal mesh ground and is exposed from the sides and above. To operate the co-planar OEW device, an AC voltage is applied between the ITO layer on the OEW chip and the metal mesh grid.

Droplet actuation of the co-planar device occurs by shining a localized visible light pattern on the OEW chip. In the dark state with no light incident on the device, the applied AC voltage drops primarily across the a-Si photoconductor layer. By shining light on the OEW device as shown in Fig. 1(b), the conductivity of the a-Si increases by 100× under the illuminated areas.

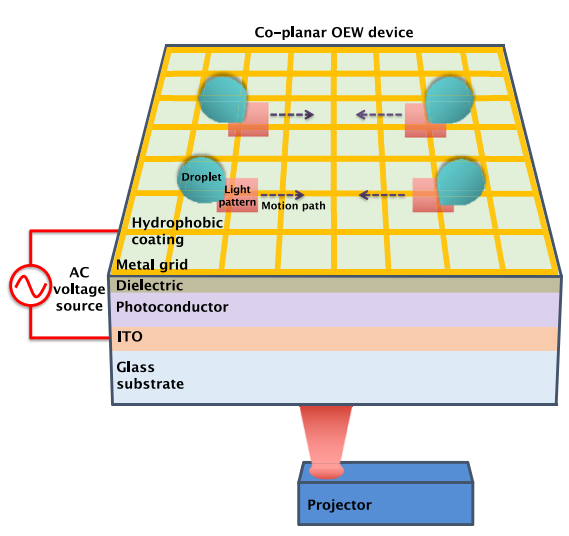

(a)

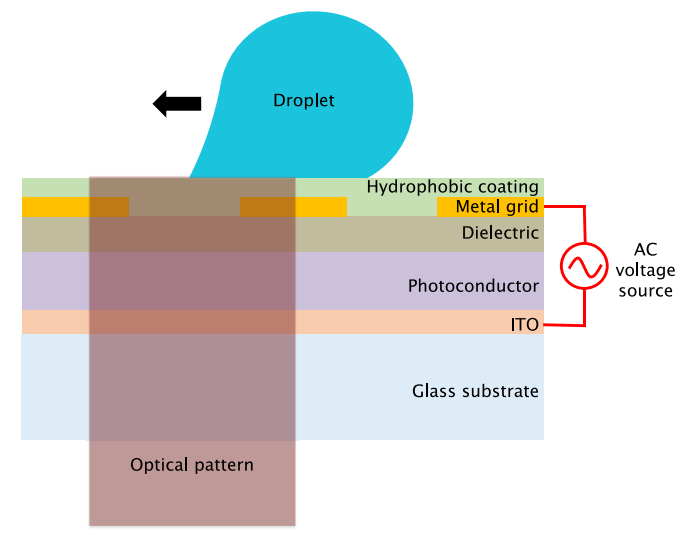

(b)

Fig. 1 (a) Top view schematic of the co-planar OEW device. (b) Cross-sectional view of the coplanar OEW device. Light is selectively illuminated on the photoconductor layer under part of a droplet's contact line to achieve an electromechanical force imbalance within the droplet. This causes the droplet to move toward the light pattern. 
Because of this increase in conductivity, the voltage drop under the illuminated areas is mostly across the dielectric layer, creating a "virtual electrode" on the device surface. Light is selectively illuminated on the photoconductor layer under part of the droplet's contact line to achieve an electromechanical force imbalance within the droplet between the light and dark states, which causes the droplet to move toward the light pattern. We can achieve droplet translation by creating a light path that the light pattern moves along on the device surface and thereby moves the droplet.

\subsection{Methods}

The co-planar OEW device is fabricated using a 280-nm ITO coated glass substrate (Thin Film Devices Inc., Anaheim, California). A 1- $\mu \mathrm{m}$ a-Si photoconductor layer is deposited (Oxford Plasmalab 80Plus) followed by a $150-\mathrm{nm} \mathrm{Al}_{2} \mathrm{O}_{3}$ layer deposited by atomic layer deposition (Picosun Sunale R150). For the integrated metal mesh ground network, a 50-nm gold metal mesh grid is deposited by e-beam evaporation (Ultek E-beam Evaporator). A thin 25-nm layer of CYTOP 809A (AGC Inc. Chemicals Company, Tokyo, Japan) is spin-coated on as a hydrophobic coating.

Aqueous droplets $(10 \mathrm{mS} / \mathrm{m}$ solution of deionized water mixed with $\mathrm{KCl})$ are introduced onto the co-planar OEW device surface from the side using a syringe needle (unless otherwise stated). 1 cst silicone oil (DMS-T01 trimethylsiloxy-terminated polydimethylsiloxane, Gelest Inc. Morrisville, PA) is used to minimize evaporation of droplets in addition to lubricating the device surface to aid droplet movement. A USB microscope camera with built-in brightfield illumination (Dino-lite Premier) is used for visualization and recording.

\subsection{Device Operation}

Device activation occurs by applying a sinusoidal AC voltage between the ITO layer and the metal mesh grid contacts, and the OEW device is generally operated at $44 \mathrm{~V}_{\mathrm{pp}}$. A consumer grade digital projector (Dell 4210X) is used to project optical patterns for OEW activation. The projected area is about $1 \mathrm{~cm} \times 1.5 \mathrm{~cm}$, and an illuminated pixel from the projector is approximately $10 \mu \mathrm{m} \times 10 \mu \mathrm{m}$ on the device. Optical patterns are generated on a computer and are outputted through the connected projector. Optical patterns for droplet movement can be composed of multiple pixels and can be customized to the size of the droplets.

\section{Circuit Model}

Figure 2 depicts the co-planar OEW cross section with each layer's corresponding circuit elements. Looking at a top view of the metal mesh grid for the co-planar OEW in Fig. 3, we define the metal line as having a width $W$ and the metal pitch between two metal lines as $P$. $A_{\text {shunt }}$ is the area underneath the metal line width that creates a shunt path. The area between metal lines is the active OEW area $A_{\mathrm{OEW}}$. $A_{\text {shunt }}$ and $A_{\mathrm{OEW}}$ are defined in terms of the metal line width $W$ and metal pitch spacing $P$ as

$$
\begin{gathered}
A_{\text {shunt }}=2 P W-W^{2}, \\
A_{\mathrm{OEW}}=(P-W)^{2} .
\end{gathered}
$$

The dielectric layers $\left(\mathrm{Al}_{2} \mathrm{O}_{3}\right.$ and the hydrophobic coating $)$ are each modeled as a capacitor $C_{\text {dielectric }}$ with a capacitance of

$$
C_{\text {dielectric }}=\frac{\varepsilon_{0} \varepsilon_{\text {dielectric }} A}{t_{\text {dielectric }}},
$$

where $A$ refers to either $A_{\mathrm{OEW}}$ or $A_{\text {shunt }}, \varepsilon_{0}$ is the permittivity of free space, $\varepsilon_{\text {dielectric }}$ is the dielectric constant, and $t_{\text {dielectric }}$ is the thickness of the dielectric layer. Its $\mathrm{AC}$ impedance $Z_{\text {dielectric }}$ operates at an $\mathrm{AC}$ frequency $\omega$ and is described as 


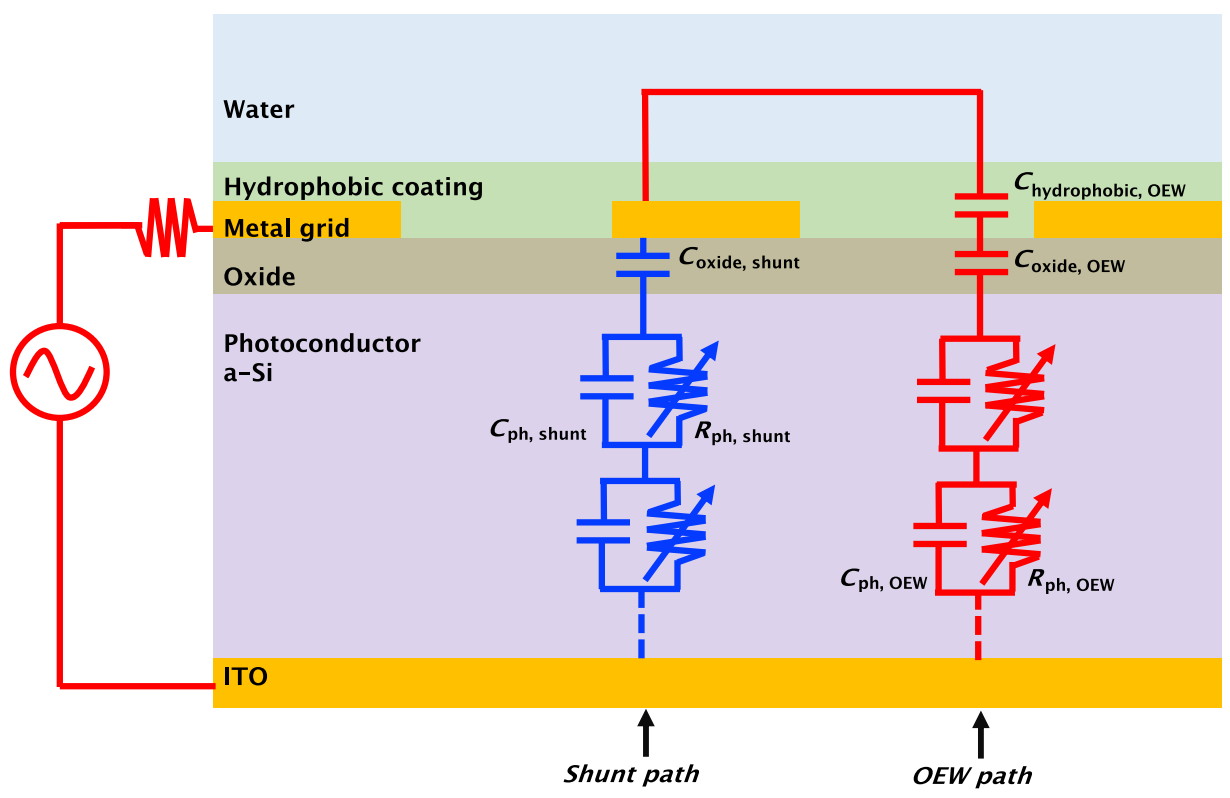

Fig. 2 Circuit model of the co-planar OEW device.

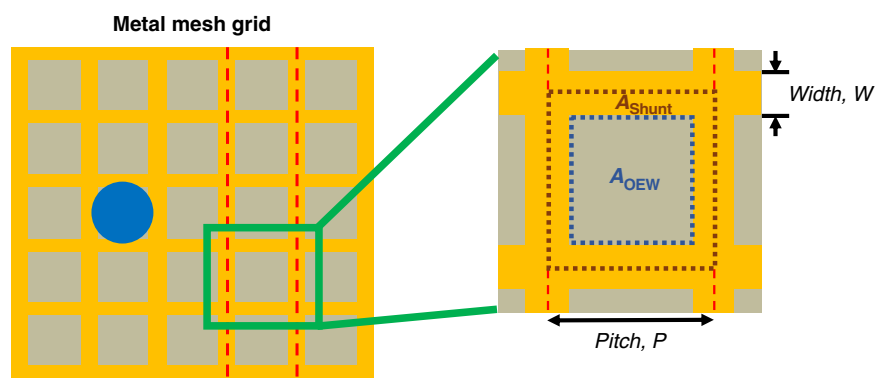

Fig. 3 Top view of the metal mesh grid geometry. The metal line width is defined as $W$. The distance between two metal lines is the pitch size $P$.

$$
Z_{\text {dielectric }}=\frac{1}{j \omega C_{\text {dielectric }}} \text {. }
$$

The a-Si photoconductor layer is modeled as an RC element with a resistance that is dependent on the light intensity incident on the device and the conductivity change between the dark and light states, which is taken from empirical values of the conductivity through the bulk of the photoconductor layer. Carriers generated by light are distributed through the photoconductor layer according to the light intensity profile and decay inversely to the absorption coefficient, exponentially from the surface. To account for the white light source that is used to produce optical patterns, the photoconductor circuit element follows a detailed analysis based on the distributed circuit model. ${ }^{36}$ We used a spectrometer (Ocean Optics Inc., Largo, FL) to measure the power density output from our experimental setup's projector white light source. For the circuit model presented, we simplified the output of our visible spectrum projector to be composed of blue $(450 \mathrm{~nm})$, green $(550 \mathrm{~nm})$, and red $(700 \mathrm{~nm})$ wavelengths. Their respective absorption coefficients were taken from previously reported measurements. ${ }^{36}$ Even when the device is in the dark state with all of the projector's pixels turned to black, there is still a minimal amount of background light to account for.

To implement the distributed circuit model for the photoconductor layer in our co-planar OEW device, we model the photoconductor layer as infinitely small slices of photoconductor 
layers with a thickness $\delta x$. Each slice is its own RC element, where the resistance $R_{\mathrm{ph}}$ and capacitance $C_{\mathrm{ph}}$ are described in Eqs. (5) and (6), respectively, as

$$
\begin{aligned}
& R_{\mathrm{ph}}=\frac{\rho(x) \delta x}{A}, \\
& C_{\mathrm{ph}}=\frac{\varepsilon_{0} \varepsilon_{\mathrm{ph}} A}{\delta x},
\end{aligned}
$$

where $\rho(x)$ is the resistivity at a certain depth $x$ and $\varepsilon_{\mathrm{ph}}$ is the dielectric constant of the photoconductor. Therefore, the AC impedance $Z_{\delta x}$ of each slice per unit area at an AC frequency $\omega$ is

$$
Z_{\delta x}(x)=\frac{\rho(x) \delta x}{A\left(1+j \omega \varepsilon_{\mathrm{ph}} \varepsilon_{0} \rho(x)\right)} .
$$

To determine the resistance, the resistivity at each depth $x$ is determined by

$$
\rho(x)=\frac{1}{q \mu_{n} \delta n(x)+q \mu_{p} \delta p(x)},
$$

where $\delta n(x)$ and $\delta p(x)$ are the electron and hole generation concentrations, respectively, due to the light at each depth $x, q$ is the electron charge, and $\mu_{n}$ and $\mu_{p}$ are the mobility of electrons and holes, respectively. $\delta n(x)$ and $\delta p(x)$ are represented by the generation rate $G_{\delta x}$ and the electron and hole lifetimes $\tau_{n}$ and $\tau_{p}$, respectively, and are defined in Eqs. (9) and (10), respectively, as

$$
\begin{aligned}
& \delta n(x)=G_{\delta x}(x) \tau_{n}, \\
& \delta p(x)=G_{\delta x}(x) \tau_{p} .
\end{aligned}
$$

The generation rate $G_{\delta x}$ is determined by the photon flux $N_{0}$ and the absorption coefficient $\alpha$ at a depth $x$ and is expressed as

$$
G_{\delta x}(x)=\alpha N_{0} \mathrm{e}^{-\alpha x} .
$$

The photon flux $N_{0}$ is determined by wavelength $\lambda$ and the power density of the light source and is described as

$$
N_{0}=\text { power density } \times\left(\frac{c}{h \lambda}\right) \text {, }
$$

where $c$ is the speed of light and $h$ is Planck's constant. In the case of using a white light source, we must account for the range of wavelengths and the power density incident on the device to determine the total carrier generation.

The total impedance of the photoconductor layer $Z_{\mathrm{ph}}$ is the integration of all infinite slices of the photoconductor through its total thickness $t$ and is expressed as

$$
Z_{\mathrm{ph}}=\int_{0}^{\mathrm{t}} Z_{\delta x}(x) \mathrm{d} x .
$$

The total impedance of the dielectric layer in the OEW region $Z_{\text {dielectric }}$ isw a summation of the impedance of the dielectric layers that contribute to the OEW force. It is expressed as

$$
Z_{\text {dielectric }_{\mathrm{OEW}}}=Z_{\text {hydrophobic }_{\mathrm{OEW}}}+Z_{\text {oxide }_{\mathrm{OEW}}}+Z_{\text {hydrophobic }_{\text {shunt }}} \text {, }
$$

where $Z_{\text {hydrophobic }_{\text {OEW }}}$ and $Z_{\text {Oxide }_{\text {OEW }}}$ are the hydrophobic coating and oxide material impedances in the OEW area, respectively, and $Z_{\text {hydrophobic }}$ shunt is the hydrophobic coating impedance in the shunt area. 
The metal grid integrated on the OEW surface introduces a shunt path, which decreases the effective voltage for electrowetting. Under the metal grid shunt area, there is a shunt capacitance from the dielectric layer and a shunt RC distributed element from the photoconductor that both contribute to the overall shunt impedance $Z_{\text {shunt }}$. We call the impedance in the active OEW area, composed of the capacitances due to the dielectric layers and RC distributed element due to the photoconductor, $Z_{\mathrm{OEW}} \cdot Z_{\text {shunt }}$ and $Z_{\mathrm{OEW}}$ are parallel to each other, so we define their parallel impedance relationship $Z_{\mathrm{OEW}+\text { shunt }}$ as

$$
Z_{\mathrm{OEW}+\text { shunt }}=Z_{\mathrm{OEW}} \| Z_{\text {shunt }} \text {. }
$$

Since the cross-sectional circuit model in Fig. 2 only accounts for one period of the active OEW area [Eq. (2)] and metal grid area [Eq. (1)], there is an additional circuit element that shares the node with the OEW path and shunt path. This element is the overall effective metal grid resistance $Z_{\text {metal }}$. The voltage at the node in between the greater metal network and one period of OEW area $V_{\mathrm{OEW}+\text { shunt }}$ is determined by

$$
V_{\mathrm{OEW}+\text { shunt }}=\frac{Z_{\mathrm{OEW}+\text { shunt }}}{Z_{\mathrm{OEW}+\text { shunt }}+Z_{\text {metal }}} V_{\text {applied }},
$$

where $V_{\text {applied }}$ is the applied AC voltage between the ITO layer and metal grid contacts. The voltage across the dielectric in the active OEW region is, therefore, described as

$$
V_{\text {dielectric }}=\frac{Z_{\text {dielectric }_{\mathrm{OEW}}}}{Z_{\text {dielectric }_{\mathrm{OEW}}}+Z_{\mathrm{ph}_{\mathrm{OEW}}}} V_{\mathrm{OEW}+\text { shunt }},
$$

where $Z_{\mathrm{ph}}$ is is the impedance of the photoconductor layer in the OEW region.

To analyze the performance of the co-planar OEW device, we derive an equation for the force per unit length that the device exerts on the contact line of the droplet and the effect of the metal mesh grid. Since for one period of the device $A_{\text {shunt }}$ is the area covered by the metal mesh and $A_{\mathrm{OEW}}$ is the area of the active OEW region, the total area for one period of the device is the active OEW area and the shunt area combined or $A_{\mathrm{OEW}}+A_{\text {shunt }}$. We call the ratio of the active OEW area over the total area as the fill factor $(\mathrm{FF})$, which is expressed as

$$
\mathrm{FF}=\frac{A_{\mathrm{OEW}}}{A_{\mathrm{OEW}}+A_{\text {shunt }}} .
$$

The shunt area does not contribute to the electrowetting force, but instead, the metal pattern shields light from the droplet and prevents the droplet from experiencing an electrowetting force . To account for the reduction in active electrowetting, we multiply the metal mesh grid FF in Eq. (18) by the force per unit length generated by OEW across the droplet due to the difference in the voltages across the dielectric layer in the light and dark states. The net force per unit length that the droplet experiences by the co-planar OEW device $F_{\text {co-planar OEW }}$ is the force per unit length under light conditions minus the force per unit length under dark conditions and is calculated using:

$$
F_{\text {co-planar OEW }}=\frac{1}{2} \frac{\varepsilon_{0} \varepsilon_{\text {dielectric }}}{t_{\text {dielectric }}}\left(V_{\text {dielectric,light }}{ }^{2}-V_{\text {dielectric,dark }}^{2}\right) \times F F,
$$

where $V_{\text {dielectric,light }}$ and $V_{\text {dielectric,dark }}$ are the voltages dropped across the dielectric layer from Eq. (17) for the light and dark states, respectively.

Figure 4 represents a surface plot model of the net force per unit length normalized to the net force per unit length of the two-plated OEW device. The normalized net force per unit length of the two-plated OEW device is set to 1 since the force is constant as it is not affected by any effects of a metal grid (since such grid does not exist). Red indicates a higher normalized net force per unit length of up to $90 \%$, and dark blue represents a low normalized net force per unit length of down to $0 \%$ or no force. The $x$ axis represents the metal pitch distance $P$, and the $y$ axis 


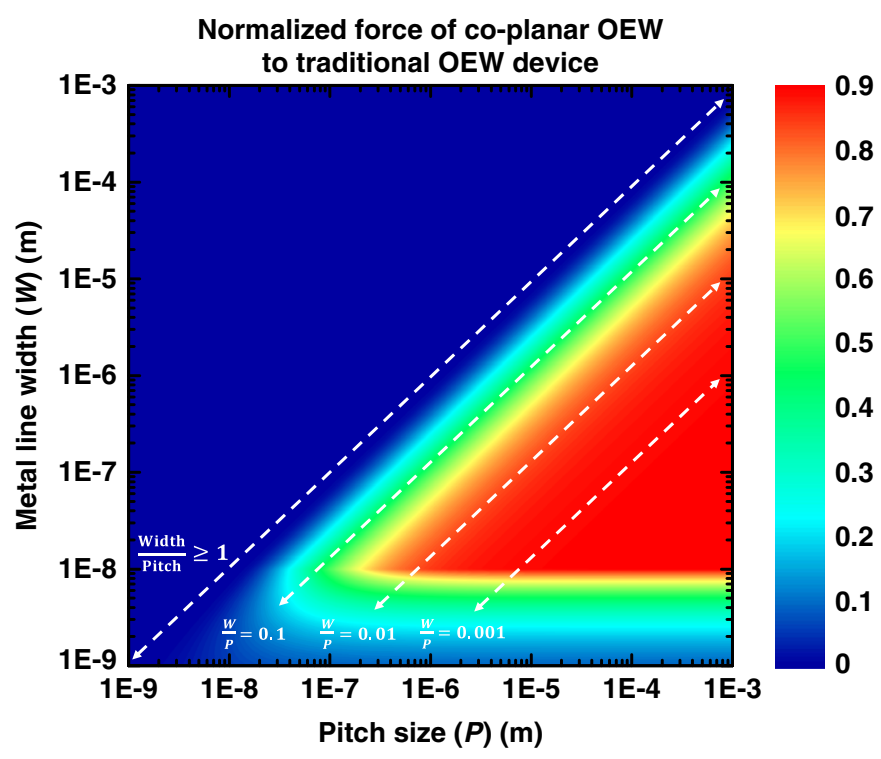

Fig. 4 Surface plot of the normalized force of the co-planar OEW device compared with the traditional two-plated OEW device as a function of metal width and pitch size. Higher OEW forces are in red, and lower OEW forces are in blue. Along fixed FF values (dotted lines), the normalized force of the co-planar OEW stays the same.

represents the metal line width $W$. The upper left triangular region of the plot is dark blue and represents a $0 \%$ net force. In this region of the model plot, the metal line width would be larger than the pitch, resulting in the whole chip being covered by metal and nullifying OEW operation due to no electrowetting effect, which is not meaningful to the study of the OEW device. We focus our discussion on the lower right triangular region of the surface plot. As the metal pitch size increases at a set metal line width, the normalized force per unit length increases for the coplanar OEW structure as more of the active OEW area is exposed. Similarly, for a given set metal pitch distance, as the metal line width decreases and becomes thinner, there is an increase in the normalized net force per unit length due to less shielding by the metal shunt area. Because of the FF effect, the normalized net force per unit length increases as $A_{\text {shunt }}$ decreases compared with the $A_{\mathrm{OEW}}$. Diagonal slices of the surface plot indicate a fixed FF ratio, meaning the ratio of $A_{\mathrm{OEW}}$ to $A_{\text {total }}$ remains the same. Along fixed FF values, the normalized force of the co-planar OEW device stays the same. The edge cases where the co-planar OEW force starts to drop off relative to the traditional OEW device is the regime where the metal line widths get significantly small and the resistance of the whole metal grid becomes significantly noticeable.

\section{Experimental Demonstrations and Results}

\subsection{Droplet Actuation Dependence on AC Frequency}

To validate the co-planar OEW model, OEW devices with different pitch sizes of 60, 100, and $300 \mu \mathrm{m}$ were fabricated with metal line widths of $3 \mu \mathrm{m}$. To measure the maximum droplet actuation speed, a $1-\mu \mathrm{L}$ water droplet was dispensed on the device surface with a 1-mm layer of $1 \mathrm{cst}$ silicone oil. A square optical pattern was programmed to translate across the device area at set speeds, which were increased until the droplet could no longer follow the optical pattern. To determine the optimal AC frequency at which to operate the co-planar OEW device, the theoretical model using a $300-\mu \mathrm{m}$ metal pitch and a $3-\mu \mathrm{m}$ metal line width in Fig. 5(a) predicts maximum force around 10 to $20 \mathrm{kHz}$. Agreeing with the model, when operating the fabricated OEW devices at $44 \mathrm{~V}_{\mathrm{pp}}$ and varying the $\mathrm{AC}$ frequencies applied from 1 to $100 \mathrm{kHz}$, droplet speeds peak around $10 \mathrm{kHz}$ as seen in Fig. 5(b). Droplet speed is used as a figure of merit to compare to force since the net force to move a droplet is a balance between the frictional forces and the actuation force generated by OEW. ${ }^{39}$ As a result, speed is proportional to force. 


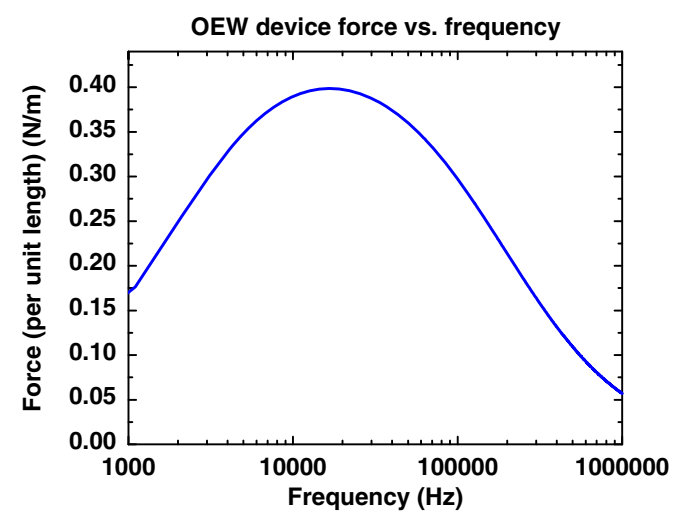

(a)

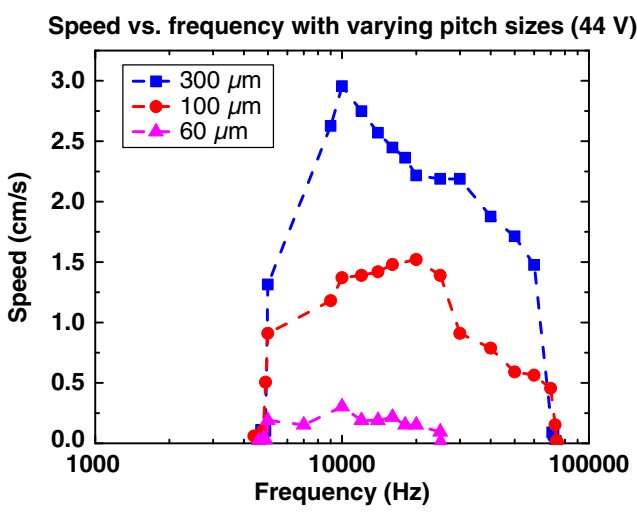

(b)

Fig. 5 (a) Co-planar OEW force versus frequency according to the co-planar OEW circuit model with a 300- $\mu \mathrm{m}$ metal pitch and a 3- $\mu \mathrm{m}$ metal line width. Predicted optimal OEW performance occurs around the 10- to $20-\mathrm{kHz}$ range. (b) Maximum droplet speed versus frequency for fabricated co-planar OEW devices with $3 \mu \mathrm{m}$ metal grid widths and various metal pitch sizes of 60,100 , and $300 \mu \mathrm{m} .1 \mu \mathrm{L} 10 \mathrm{mS} / \mathrm{m}$ aqueous droplets were dispensed and actuated to find the maximum speeds that could be achieved on these devices across a frequency range of 1 to $100 \mathrm{kHz}$ with an applied $A C$ voltage of $44 \mathrm{~V}_{\mathrm{pp}}$.

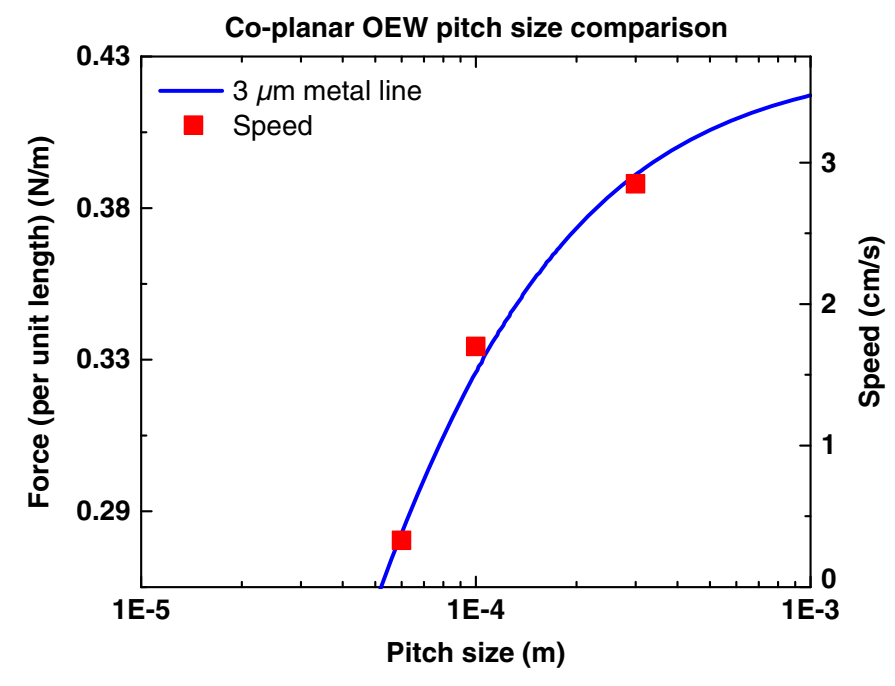

Fig. 6 Comparison of the theoretical force (left axis) and the measured maximum droplet speeds (right axis) for co-planar OEW devices with various pitch sizes $(60,100$, and $300 \mu \mathrm{m})$. To measure the maximum speeds of $1 \mu \mathrm{L}$ aqueous droplets, a bias voltage of $44 \mathrm{~V}_{\mathrm{pp}}$ at $10 \mathrm{kHz}$ was applied to fabricated co-planar OEW devices with a 3- $\mu \mathrm{m}$ metal line width and 300- $\mu \mathrm{m}$ pitch size.

For the 60,100, and $300 \mu \mathrm{m}$ metal line pitches, maximum droplet speeds of $0.3,1.5$, and $2.9 \mathrm{~cm} / \mathrm{s}$, respectively, at $44 \mathrm{~V}_{\mathrm{pp}}$ and $10 \mathrm{kHz}$ were achieved. The maximum droplet speeds attained experimentally for the varying metal pitch designs follow a similar trend to the expected peak forces derived from the co-planar OEW model as shown in Fig. 6. The maximum droplet speed of $2.9 \mathrm{~cm} / \mathrm{s}$ on the co-planar OEW device at $44 \mathrm{~V}_{\mathrm{pp}}$ and $10 \mathrm{kHz}$ is faster than that of the $1.5-\mathrm{cm} / \mathrm{s}$ speeds achieved on the traditional two-plated OEW devices ${ }^{34}$ at the same actuation voltage and frequency. The increase in speed for co-planar OEW devices is in part due to the reduced shear friction contribution by lack of a top cover. The increased droplet speeds achieved on the co-planar device demonstrate the potential to process droplets faster with higher throughput. 


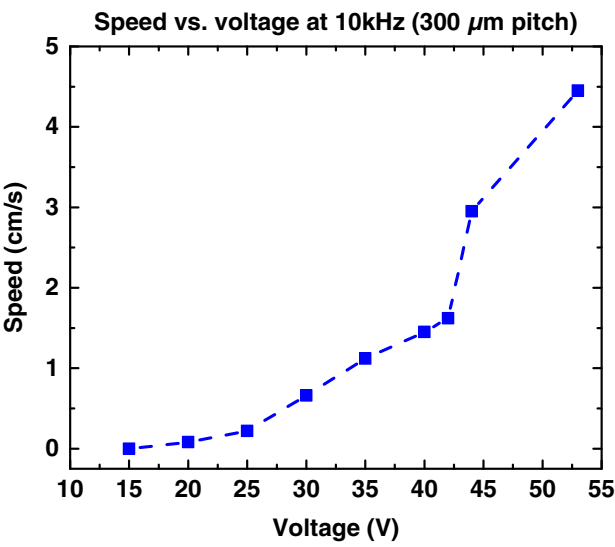

(a)

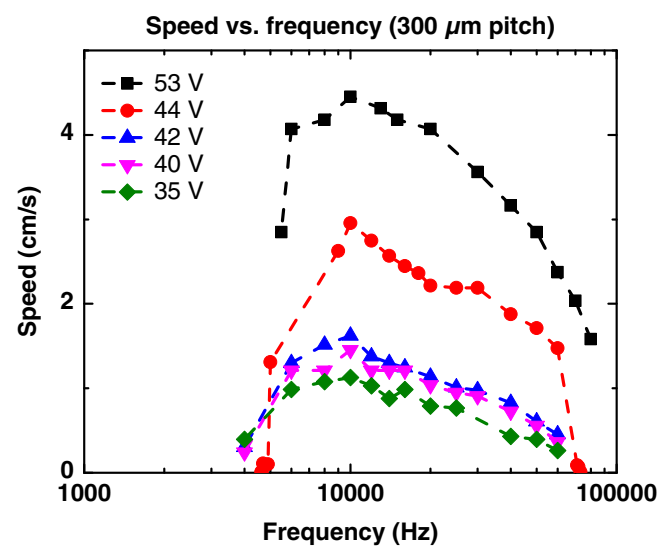

(b)

Fig. 7 (a) Maximum droplet speed versus voltage for a $1-\mu \mathrm{L}$ droplet at $10 \mathrm{kHz}$ frequency on a coplanar OEW device with a 3- $\mu \mathrm{m}$ metal line width and 300- $\mu \mathrm{m}$ pitch size. (b) Maximum droplet speed versus frequency for a $1-\mu \mathrm{L}$ droplet at various applied voltages (35 to $53 \mathrm{~V}_{\mathrm{pp}}$ ) on a co-planar OEW device with a $3-\mu \mathrm{m}$ metal line width and $300-\mu \mathrm{m}$ pitch size.

\subsection{Droplet Actuation Dependence on Voltage}

The force per unit length of the droplet's moving contact line is highly dependent on the voltage difference across the dielectric layer with and without light incident on the OEW device. Increasing the voltage applied to our OEW device will generate a greater OEW force and faster droplet translation. However, lower voltage operation is ideal for avoiding dielectric breakdown, safer operation, and being more energy efficient. Using the 300- $\mu \mathrm{m}$ pitch size OEW devices and setting the operational frequency to the peak value at $10 \mathrm{kHz}$, droplet speeds were measured as a function of applied voltage as shown in Fig. 7(a). Droplet speeds increased to a maximum speed of $4.5 \mathrm{~cm} / \mathrm{s}$ at $53 \mathrm{~V}_{\mathrm{pp}}$, which is over $2 \times$ the maximum droplet speed achieved on the two-plated OEW device of $2.0 \mathrm{~cm} / \mathrm{s}$ at $52 \mathrm{~V}_{\mathrm{pp}} .{ }^{34}$ Droplet movement was still realized on the co-planar OEW device with an applied voltage as low as $16 \mathrm{~V}_{\mathrm{pp}}$ at a speed of $0.2 \mathrm{~cm} / \mathrm{s}$. Figure $7(\mathrm{~b})$ demonstrates the frequency dependence of the co-planar OEW device for various applied voltages from 35 to $53 \mathrm{~V}$. Peak speeds were consistently achieved around $10 \mathrm{kHz}$, further confirming the co-planar OEW circuit model.

\subsection{Droplet Actuation Dependence on Light Intensity}

The ability to further reduce the optical power used while maintaining reliable droplet movement is explored. Low light intensity operation is beneficial for a more energy efficient system and for light and temperature sensitive applications. Previous generations of OEW and their experimental setups utilized a monochromatic 5-mW laser pointer to manipulate droplets with an optical intensity of $250 \mathrm{~W} / \mathrm{cm}^{2,29-33}$ To reduce the light intensity, a commercially available DLP projector replaced the use of a higher-powered laser. ${ }^{36}$ The projector light source (Dell 4210X) used to measure the co-planar OEW device is equipped with a white light source and a measured intensity of $1.5 \mathrm{~W} / \mathrm{cm}^{2}$ at our device surface.

To study the lower limits of optical intensities that can be used to maintain reliable droplet control, neutral density (ND) filters were used between the projector output and the OEW device to reduce the transmission of light evenly across the visible spectrum of light emitted by the projector bulb. The speed of a $1-\mu \mathrm{L}$ droplet traveling across the OEW device for various light intensities from $100 \%$ transmission (no filter) down to $3 \%$ transmission was measured across a range of frequencies from 1 to $100 \mathrm{kHz}$ at $53 \mathrm{~V}_{\mathrm{pp}}$ as graphed in Fig. 8. Maximum speeds were consistently attained around $10 \mathrm{kHz}$ regardless of the light intensity incident on the OEW device. Droplets were still consistently and reliably able to move across a large range of frequencies on the device surface with light intensities as low as $5 \%$ transmission or $0.075 \mathrm{~W} / \mathrm{cm}^{2}$. At this intensity of light, peak droplet speeds of $1.9 \mathrm{~cm} / \mathrm{s}$ were achieved. Even at $16 \%$ transmission 


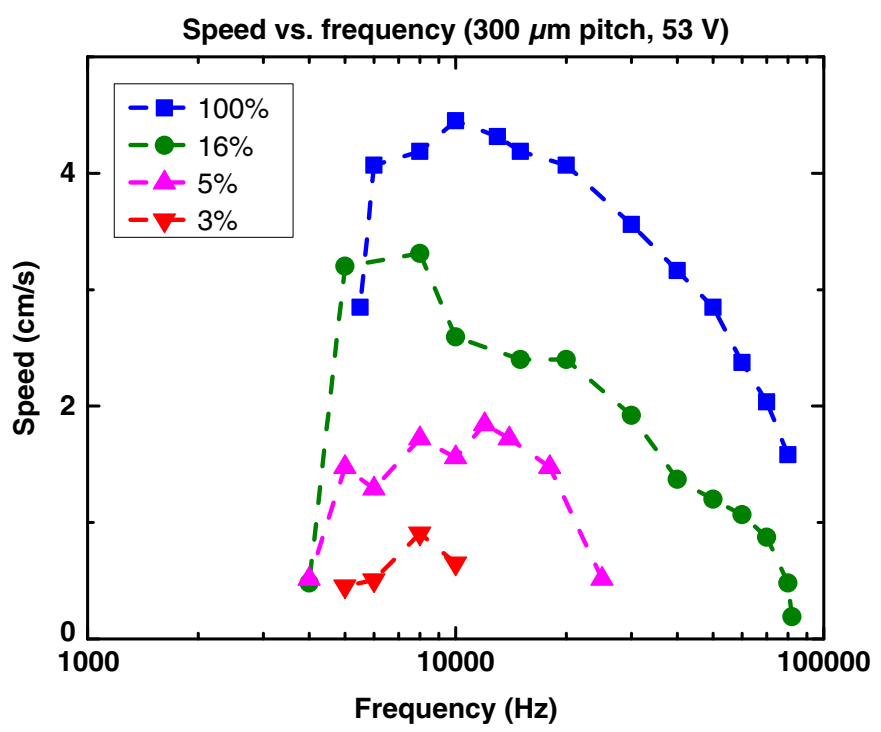

Fig. 8 Maximum droplet speed versus frequency at various optical pattern light intensities for a $1-\mu \mathrm{L}$ droplet at an applied voltage of $53 \mathrm{~V}_{\mathrm{pp}}$. ND filters with various optical densities were used to reduce the incident light intensity from $100 \%$ (no filter) down to $3 \%$ light transmission on the OEW device surface.

or $0.24 \mathrm{~W} / \mathrm{cm}^{2}$, peak droplet speeds of $3.3 \mathrm{~cm} / \mathrm{s}$ at $10 \mathrm{kHz}$ were achieved. The co-planar OEW device benefits from increased efficiency compared with the two-plated OEW device as it can achieve the same droplet speeds with a 95\% reduction in light intensity.

\subsection{Parallel Droplet Movement}

A droplet can move on the OEW surface as long as the droplet is in contact with the active OEW area and a metal mesh ground line. Because of this, OEW has the ability to move droplets in parallel around the chip in not only a programmed rectangular path but also a circular path as shown in Figs. 9(a) and 9(b), respectively. This demonstrates the ability of the droplet to freely move in any path desired around the two-dimensional plane. To accommodate more droplets on the surface of the chip, additional light patterns are illuminated on the photoconductor layer by the optical projector setup by turning various pixels on and off under the droplets. The size and movement of each light pattern can be resized and reshaped for different droplet sizes and paths.

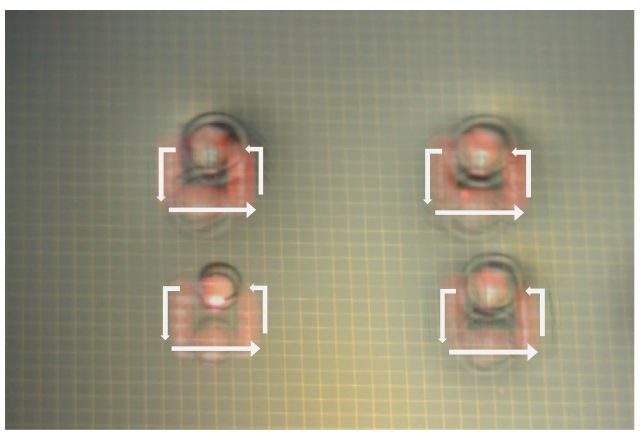

(a)

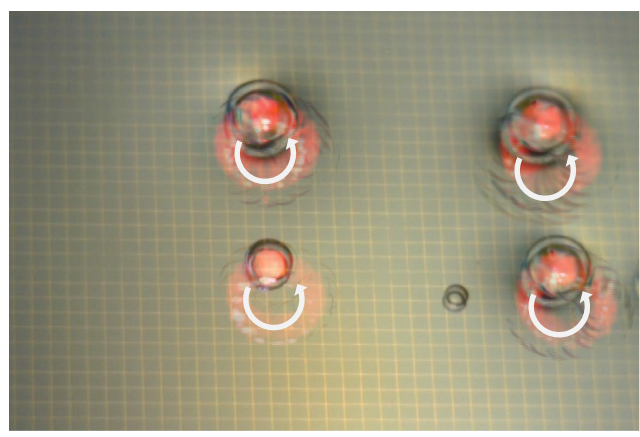

(b)

Fig. 9 Stacked images of parallel movement of droplets on the co-planar OEW device. Four distinct $10 \mathrm{mS} / \mathrm{m}$ aqueous droplets move in parallel on a 3- $\mu \mathrm{m}$ metal line width and 300- $\mu \mathrm{m}$ metal pitch sized OEW device following a (a) rectangular motion path and (b) circular motion path. Droplets were actuated at $44 \mathrm{~V}_{\mathrm{pp}}$ at $10 \mathrm{kHz}$. 

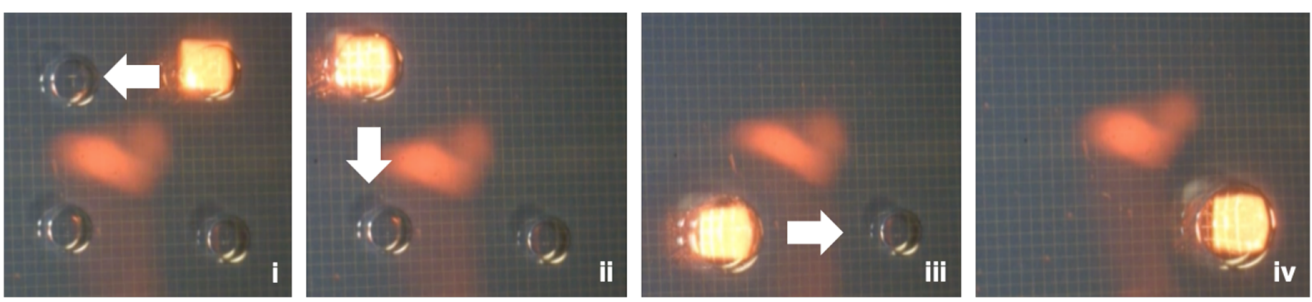

Fig. 10 Sequential merging of four $1 \mu \mathrm{L}$ droplets on the 3- $\mu \mathrm{m}$ metal line width and $300-\mu \mathrm{m}$ pitch size co-planar OEW device. The OEW device is biased at $44 \mathrm{~V}_{\mathrm{pp}}$ and $10 \mathrm{kHz}$.

\subsection{Sequential Droplet Merging}

Droplets can also merge with other droplets by moving two (or more) droplets toward each other. As shown in Fig. 10, multiple droplets can be sequentially moved around to merge with each other. This demonstrates not only that droplets can be merged together but also that the merged droplet now contains a greater combined volume and can be moved with the same light pattern. OEW's flexibility allows for a wide range of droplet sizes and motion paths to be moved around on its surface without needing to change the fundamental device design.

\subsection{Unique Droplet Mixtures}

Droplets can also be moved and merged in parallel to create unique mixtures. In Fig. 11, there are six $1 \mu \mathrm{L}$ aqueous droplets, each containing a unique dye color of either red, yellow, or blue (McCormick Food Coloring, Baltimore, MD). The column on the left contains droplets (from top to bottom) with red, blue, and yellow dyed $1 \mu \mathrm{L}$ aqueous droplets, and the column on the right contains $1 \mu \mathrm{L}$ droplets (from top to bottom) with blue, yellow, and red dyed droplets. The two droplets from the same row (one droplet from the left column and one droplet from the right column) move at the same time toward each other. The two droplets eventually meet in the middle and combine to form a single droplet of a combined $2 \mu \mathrm{L}$ volume. This occurs for all three rows simultaneously to form a resulting single column made out of three droplets with the resulting mixed colors of purple (red mixed with blue), green, (blue mixed with yellow), and orange (red mixed with yellow). The mixing of the colors demonstrates not only the ability to

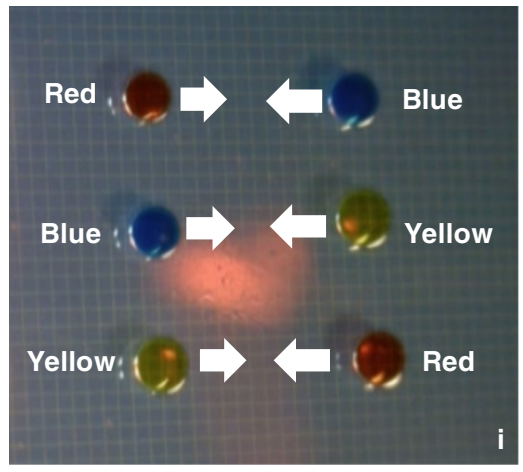

Unmixed droplets with dye

(a)

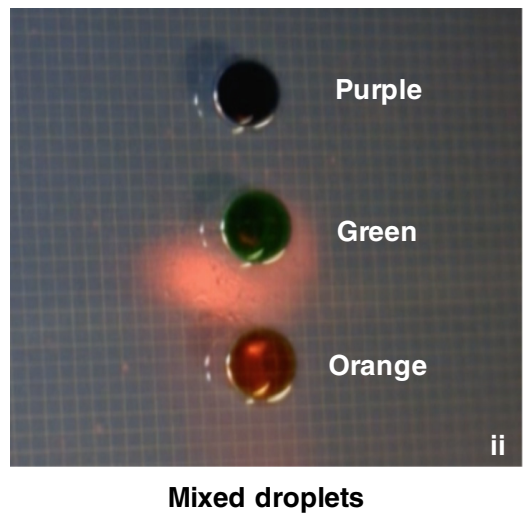

(b)

Fig. 11 Droplets on the co-planar OEW device are moved and mixed in parallel to create unique droplet mixtures. (a) Two columns of droplets made up of $1 \mu \mathrm{L}$ aqueous droplets containing different colored food dyes. The left column contains droplets from top to bottom of red, blue, and yellow coloration. The right column contains droplets from top to bottom of blue, yellow, and red coloration. (b) Droplets from both columns are moved toward the center in parallel to mix together to create one column of mixed colored droplets of purple (red + blue), green (blue + yellow), and orange (yellow + red). 
merge droplets but also the ability of the OEW device to support a variety of unique droplets and mixtures in parallel on chip for further processing. Each discrete droplet on the OEW device can serve as its own reaction chamber.

\subsection{Droplet-on-Demand System Integration}

One of the main advantages of the co-planar OEW device with its open top is the ability to integrate the device with a wider range of input/output capabilities. Traditional OEW devices with a conductive top plate require that input/output configurations be integrated from the sides of the device in between the OEW chip and the top plate. Although input/output configurations from the sides are feasible, it can be more challenging and limiting than input/output capabilities on devices without a top cover.

To utilize one of the co-planar OEW device's main benefits of having more accessible input/ output configurations for downstream applications, the ability to dispense droplets from above is demonstrated. A droplet-on-demand dispensing system was acquired (Microfab Technologies, Texas). The dispensing unit is composed of a piezoelectric nozzle driver that controls the volume of liquid dispensed by a proprietary controller box and software via a computer. Nozzles supplied by Microfab come in a range of diameters and are interchangeable. Reservoirs of the sample solutions are connected to the nozzle and backflow of the liquid is controlled by the pressure control box. A USB camera (Omron Sentech, Kyoto, Japan) is aligned below the output nozzle in line with a strobe light to characterize the droplet as viewed via a computer monitor and to adjust dispensing settings of the drive controller.

A 50- $\mu \mathrm{m}$ diameter size nozzle was used to dispense individual drops with volumes of $65 \mathrm{pL}$ at a $120-\mathrm{Hz}$ frequency from the droplet-on-demand system. We can customize the total volume of a droplet that is dispensed on a chip by accumulating these individual $65 \mathrm{pL}$ drops to form a larger droplet volume.

\subsection{1 "Cal" logo demonstration}

In Fig. 12, $65 \mathrm{~nL}$ droplets made up of 1000 accumulated $65 \mathrm{pL}$ drops were dispensed on the right side of the OEW working area and moved into position via an optical pattern to form the University of California, Berkeley's Cal logo. Thirty droplets in total were created and moved into formation. This demonstration shows the capability for integration from above and the ability for droplets to move freely around the co-planar OEW device plane.

\subsubsection{Droplet array formation}

One benefit of microfluidics is the ability not only to miniaturize the reaction volumes used but also to increase the number and throughput of droplets that can be processed at the same time. We integrate the droplet dispensing technique from above by our droplet-on-demand dispensing machine with our OEW device to dispense droplets and position these droplets into an orderly array. Each location with an individual droplet acts as an individual reaction volume. In Fig. 13, an array of 100 droplets, each having a volume of $65 \mathrm{~nL}$, were dispensed from above. Each $65 \mathrm{~nL}$
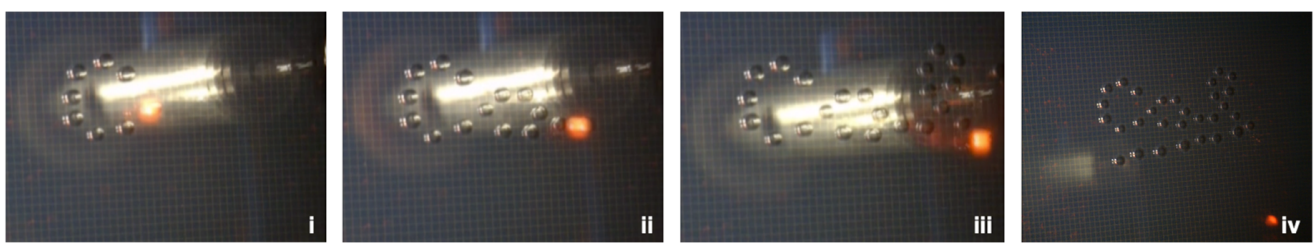

Fig. 12 Snapshots of droplets moved to form the University of Berkeley, California Cal logo. $65 \mathrm{~nL}$ total volume droplets dispensed by a droplet-on-demand system from above to the right side on the co-planar OEW device's active region and positioned on the device surface via OEW to form the Cal logo. The 3- $\mu \mathrm{m}$ metal line width and 300- $\mu \mathrm{m}$ metal pitch size OEW device was actuated at $44 \mathrm{~V}_{\mathrm{pp}}$ and $10 \mathrm{kHz}$. 

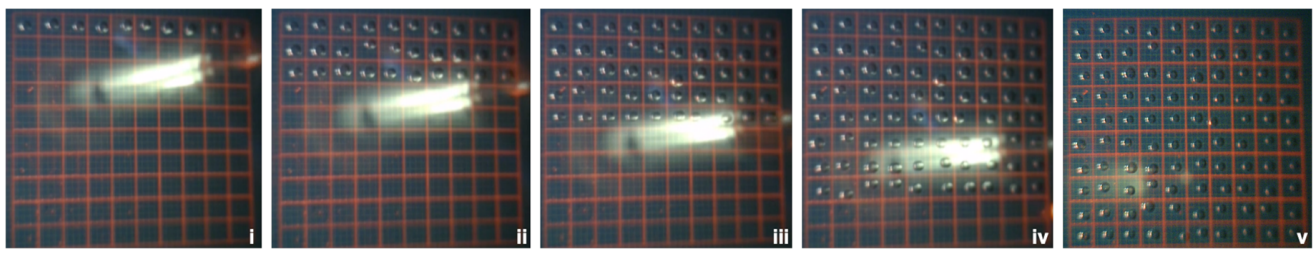

Fig. 13 Formation of a $10 \times 10$ droplet array on the co-planar OEW device. $65 \mathrm{~nL}$ final volume droplets were dispensed by a droplet-on-demand system from above to an area on the right side of the co-planar OEW device with a 3- $\mu \mathrm{m}$ metal line width and 300- $\mu \mathrm{m}$ metal pitch size. Droplets were then moved by OEW and placed into position row by row. The red grid-like pattern is an optical pattern produced by the projector system for visual purposes. The OEW device was biased at $44 V_{\mathrm{pp}}$ and $10 \mathrm{kHz}$.

droplet dispensed was composed of 1000 drops of $65 \mathrm{pL}$, each at a frequency of $120 \mathrm{~Hz}$. In this array, the 100 droplets were fashioned into a $10 \times 10$ square array. Droplets were dispensed by accumulating each $65 \mathrm{~nL}$ droplet volume on an area to the side of the array and utilizing light patterns to move the droplet to a specified location via OEW. The red grid outline encompassing each droplet was created by the light projector from below shining a faint red optical pattern and aided in visualizing and organizing the $10 \times 10$ array.

We can scale up the number of droplets that can be formed and moved to cover the area of our device. The co-planar OEW chip used in these demonstrations has an active OEW area of size $25 \mathrm{~mm} \times 25 \mathrm{~mm}$. Figure 14 demonstrates the ability to increase our droplet array to fill up our whole OEW surface area with 400 droplets organized in a $20 \times 20$ array. Each droplet dispensed was composed of $150065 \mathrm{pL}$ drops at a frequency $120 \mathrm{~Hz}$ to make a cumulative droplet size of $100 \mathrm{~nL}$. The droplet volume for the $20 \times 20$ array was different than the $10 \times 10$ array to demonstrate how droplet volumes can vary for each experiment depending on the need. Droplets for the $20 \times 20$ array were dispensed and positioned using a combination of two methods: (1) dispensing individual drops from above and accumulating the full $100 \mathrm{~nL}$ droplet on the side of the array and using light patterns via OEW to move the droplet to a specified location and (2) dispensing droplets from directly above the specified target location and using OEW to
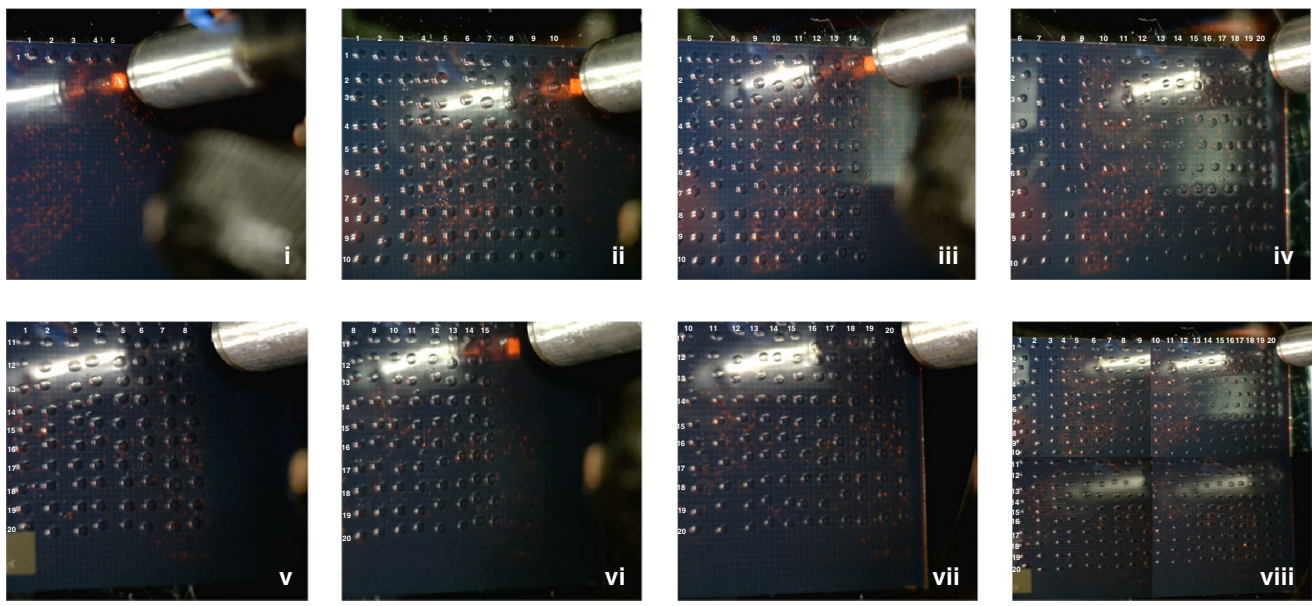

Fig. 14 Formation of a $20 \times 20$ droplet array on the co-planar OEW device. $100 \mathrm{~nL}$ droplets were dispensed and moved via OEW into a $20 \times 20$ array formation on the $3-\mu \mathrm{m}$ metal line width and 300- $\mu \mathrm{m}$ metal pitch size device. The OEW device was biased at $44 \mathrm{~V}_{\mathrm{pp}}$ and $10 \mathrm{kHz}$. The start of the $20 \times 20$ array formation is pictured in (i). Snapshots of intermediate progress of the droplet array are captured through successful droplet placement in: (ii) rows 1 to 10 and columns 1 to 10, (iii) rows 1 to 10 and columns 6 to 14, (iv) rows 1 to 10 and columns 6 to 20, (v) rows 11 to 20 and columns 1 to 8 , (vi) rows 11 to 20 and columns 8 to 15, and (vii) rows 11 to 20 and columns 10 to 20. The completed $20 \times 20$ array is shown in (viii) by stitching images from each completed quadrant of the OEW device taken by the brightfield camera. 
efficiently collect the individual drops to form the cumulative $100 \mathrm{~nL}$ droplet. The second method has an advantage when droplets are tightly spaced together.

These demonstrations show how the co-planar OEW's open top design is advantageous for macro- to microsystem integration. The co-planar OEW device presents itself as a candidate for downstream applications. Established research and industry tools that output droplets containing products of interest can be directly integrated with the co-planar OEW device for further processing.

\section{Conclusion}

We have presented the theory, design, and demonstration of a co-planar OEW device. The coplanar OEW device replaces the top cover electrode from previous generations of OEW devices with an integrated metal mesh on the OEW surface. Demonstrations of fabricated co-planar OEW devices confirm our theoretical model while also improving performance with faster droplet speeds of up to $4.5 \mathrm{~cm} / \mathrm{s}$, a more than $2 \times$ improvement. Basic droplet manipulations such as the merging of multiple droplets, the ability to accommodate and move droplets of differing volumes, and individual droplets operating in parallel freely around the two-dimensional device plane are still feasible. Due to its open top configuration, the co-planar OEW device introduces greater possibilities for macro- to microfluidic system integration. By integrating a droplet-ondemand dispensing system with our co-planar OEW device to generate droplets of varying volumes from above, we have successfully demonstrated the ability to collect and position the droplets on the device surface. Droplets arranged in arrays of up to $20 \times 20$ have been accomplished on our device. The demonstrations presented in this paper show how the co-planar OEW device has the potential to automate and scale down biological and chemical processes that currently utilize conventional well plates, resulting in reduced volumes of solutions, reduced manual labor, and increased processing throughput. With faster droplet speeds and more efficient operational conditions, the concept and realization of this co-planar OEW device further expand the benefits of OEW as a versatile and flexible light-actuated digital microfluidics platform.

\section{Acknowledgments}

The authors would like to thank the Berkeley Marvell Nanolab, where the OEW devices were fabricated. This work was supported in part by the Berkeley Sensor and Actuator Center (BSAC).

\section{References}

1. M. G. Pollack, R. B. Fair, and A. D. Shenderov, "Electrowetting-based actuation of liquid droplets for microfluidic applications," Appl. Phys. Lett. 77(11), 1725-1726 (2000).

2. S. K. Cho, H. Moon, and C. J. Kim, "Creating, transporting, cutting, and merging liquid droplets by electrowetting-based actuation for digital microfluidic circuits," $J$. Microelectromech. Syst. 12(1), 70-80 (2003).

3. S. Y. Teh et al., "Droplet microfluidics," Lab Chip 8(2), 198-220 (2008).

4. H. H. Shen et al., "EWOD microfluidic systems for biomedical applications," Microfluid. Nanofluid. 16(5), 965-987 (2014).

5. N. T. Nguyen, S. T. Wereley, and S. A. M. Shaegh, Fundamentals and Applications of Microfluidics, Artech House, Norwood (2019).

6. V. B. Kothamachu, S. Zaini, and F. Muffatto, "Role of digital microfluidics in enabling access to laboratory automation and making biology programmable," SLAS Technol. 25(5), 411-426 (2020).

7. J. Li and C. J. Kim, "Current commercialization status of electrowetting-on-dielectric (EWOD) digital microfluidics," Lab Chip 20(10), 1705-1712 (2020).

8. S. C. Shih et al., "A versatile microfluidic device for automating synthetic biology," ACS Syn. Biol. 4(10), 1151-1164 (2015).

9. L. Mazutis et al., "Single-cell analysis and sorting using droplet-based microfluidics," Nat. Protoc. 8(5), 870-891 (2013). 
10. S. Köster et al., "Drop-based microfluidic devices for encapsulation of single cells," Lab Chip 8(7), 1110-1115 (2008).

11. A. R. Wheeler et al., "Digital microfluidics with in-line sample purification for proteomics analyses with MALDI-MS," Anal. Chem. 77(2), 534-540 (2005).

12. M. Hosokawa et al., "Massively parallel whole genome amplification for single-cell sequencing using droplet microfluidics," Sci. Rep. 7(1), 5199 (2017).

13. R. Sista et al., "Development of a digital microfluidic platform for point of care testing," Lab Chip 8(12), 2091-2104 (2008).

14. T. Nisisako, T. Torii, and T. Higuchi, "Droplet formation in a microchannel network," Lab Chip 2(1), 24-26 (2002).

15. A. S. Utada et al., "Monodisperse double emulsions generated from a microcapillary device," Science 308(5721), 537-541 (2005).

16. T. Thorsen et al., "Dynamic pattern formation in a vesicle-generating microfluidic device," Phys. Rev. Lett. 86(18), 4163 (2001).

17. D. N. Adamson et al., "Production of arrays of chemically distinct nanolitre plugs via repeated splitting in microfluidic devices," Lab Chip 6(9), 1178-1186 (2006).

18. J. Clausell-Tormos et al., "Droplet-based microfluidic platforms for the encapsulation and screening of mammalian cells and multicellular organisms," Chem. Biol. 15(5), 427-437 (2008).

19. L. Mazutis et al., "Single-cell analysis and sorting using droplet-based microfluidics," Nat. Protoc. 8(5), 870-891 (2013).

20. H. Zhang, H. Chang, and P. Neuzil, "DEP-on-a-chip: dielectrophoresis applied to microfluidic platforms," Micromachines 10(6), 423 (2019).

21. H. A. Pohl, Dielectrophoresis: The Behavior of Neutral Matter in Nonuniform Electric Fields, Cambridge Monographs on Physics, Cambridge University Press, Cambridge/ New York (1978).

22. A. Wixforth et al., "Acoustic manipulation of small droplets," Anal. Bioanal. Chem. 379(7-8), 982-991 (2004).

23. I. Leibacher, P. Reichert, and J. Dual, "Microfluidic droplet handling by bulk acoustic wave (BAW) acoustophoresis," Lab Chip 15(13), 2896-2905 (2015).

24. T. Franke et al., "Surface acoustic wave (SAW) directed droplet flow in microfluidics for PDMS devices," Lab Chip 9(18), 2625-2627 (2009).

25. H. Tsuchiya et al., "On-chip polymerase chain reaction microdevice employing a magnetic droplet-manipulation system," Sens. Actuators B 130(2), 583-588 (2008).

26. Y. Wang, Y. Zhao, and S. K. Cho, "Efficient in-droplet separation of magnetic particles for digital microfluidics," J. Micromech. Microeng. 17(10), 2148 (2007).

27. M. A. Gijs, "Magnetic bead handling on-chip: new opportunities for analytical applications," Microfluid. Nanofluid. 1(1), 22-40 (2004).

28. M. Abdelgawad and A. R. Wheeler, "The digital revolution: a new paradigm for microfluidics," Adv. Mater. 21(8), 920-925 (2009).

29. P. Y. Chiou et al., "Light actuation of liquid by optoelectrowetting," Sens. Actuators A 104(3), 222-228 (2003).

30. H. Song, D. L. Chen, and R. F. Ismagilov, "Reactions in droplets in microfluidic channels," Angew. Chem. Int. Ed. 45(44), 7336-7356 (2006).

31. E. C. Yeh et al., "Self-powered integrated microfluidic point-of-care low-cost enabling (SIMPLE) chip," Sci. Adv. 3(3), e1501645 (2017).

32. P. Y. Chiou, Z. Chang, and M. C. Wu, "Droplet manipulation with light on optoelectrowetting device," J. Microelectromech. Syst. 17(1), 133-138 (2008).

33. P. Y. Chiou, S. Y. Park, and M. C. Wu, "Continuous optoelectrowetting for picoliter droplet manipulation," Appl. Phys. Lett. 93(22), 221110 (2008).

34. S. N. Pei et al., "Light-actuated digital microfluidics for large-scale, parallel manipulation of arbitrarily sized droplets," in IEEE 23rd Int. Conf. Micro Electro Mech. Syst., IEEE, pp. 252-255 (2010).

35. J. K. Valley et al., "A unified platform for optoelectrowetting and optoelectronic tweezers," Lab Chip 11(7), 1292-1297 (2011). 
36. S. N. Pei et al., "Distributed circuit model for multi-color light-actuated opto-electrowetting microfluidic device," J. Lightwave Technol. 33(16), 3486-3493 (2015).

37. H. S. Chuang, A. Kumar, and S. T. Wereley, "Open optoelectrowetting droplet actuation," Appl. Phys. Lett. 93(6), 064104 (2008).

38. S. Y. Park, M. A. Teitell, and E. P. Chiou, "Single-sided continuous optoelectrowetting (SCOEW) for droplet manipulation with light patterns," Lab Chip 10(13), 1655-1661 (2010).

39. V. Bahadur and S. V. Garimella, "An energy-based model for electrowetting induced droplet actuation," J. Micromech. Microeng. 16(8), 1494 (2006).

Jodi Loo received her $\mathrm{BS}$ degree and $\mathrm{PhD}$ in electrical engineering and computer sciences from the University of California, Berkeley, CA, USA, in 2014 and 2020, respectively. Her research interests include optofluidics, optoelectronics, and microfluidic systems. She was the recipient of the NSF Graduate Research Fellowship.

Shao Ning Pei received his BS degree in electrical engineering from the University of Michigan, Ann Arbor, MI, USA, in 2009 and his MS degree and PhD in electrical engineering and computer sciences from the University of California, Berkeley, CA, USA, in 2011 and 2015, respectively. His research interests include microfluidics, optofluidics, single-cell analysis, and microelectromechanical systems.

Ming C. Wu received his PhD from the University of California, Berkeley in 1988. He is a Nortel distinguished professor of electrical engineering and computer sciences at the UC Berkeley and a co-director of the Berkeley Sensor and Actuator Center. Before joining the Berkeley faculty, he was at AT\&T Bell Labs and UCLA. His research focuses on MEMS, optofluidics, optoelectronics, and nanophotonics. He is an IEEE and OSA fellow. He received several awards including the Robert Bosch MEMS award from IEEE. 